\title{
Consumo de álcool entre adolescentes do sexo feminino
}

\section{Alcohol use among females adolescents}

\author{
Gilda Pulcherio ${ }^{1}$, Tamires Bastos², Marlene Strey33, Raquel de Boni ${ }^{4}$ \\ 1 Instituto de Prevenção e Pesquisa em Álcool e outras Dependências (IPPAD), Porto Alegre/RS, Brasil. \\ 2 Centro de Pesquisa em Álcool e Drogas/Universidade Federal do Rio Grande do Sul (CPAD/UFRGS), Porto Alegre/RS, Brasil. \\ ${ }_{3}^{3}$ Programa de Pós-Graduação em Psicologia da Pontifícia Universidade Católica do Rio Grande do Sul (PUCRS), Porto Alegre/RS, Brasil. \\ 4 UFRGS, Porto Alegre/RS, Brasil.
}

Recebido: 26/11/2010 - Aceito: 4/4/2011

Pulcherio G, et al. / Rev Psiq Clín. 2011;38(5):209-10

É conhecido que o uso e o abuso de substâncias psicoativas pelas mulheres têm crescido no mundo ocidental, o mesmo ocorrendo entre as brasileiras ${ }^{1}$. Esse consumo pode acarretar inúmeras consequências e durante a gestação implica risco grave à saúde materna e fetal, favorecendo maior número de abortos, partos prematuros, retardo cognitivo, crescimento restrito do concepto, entre outras ${ }^{2}$.

No caso particular do álcool, a prevalência de síndrome alcoólica fetal (SAF) chega a $6 \%$ nos filhos de mães alcoolistas ${ }^{3}$, e mesmo naquelas crianças que não preenchem critérios para a síndrome, consequências comportamentais e emocionais tais como impulsividade, promiscuidade, problemas de socialização e de comunicação podem ser observadas mais frequentemente, quando comparadas com crianças da mesma idade que não foram expostas. Também para a mãe a exposição ao álcool traz agravos físicos, que incluem associação com transmissão de doenças sexualmente transmissíveis, e psicológicos, não havendo níveis seguros conhecidos para o consumo do álcool durante a gestação4.

O V Levantamento Nacional sobre o Consumo de Drogas Psicotrópicas ${ }^{5}$, que entrevistou estudantes do ensino fundamental e médio, mostrou que $80,8 \%$ dos estudantes entre 16 e 18 anos já consumiram álcool ao longo da vida. A precocidade da experimentação surge com a prevalência de $41,2 \%$ para as crianças entre 10 e 12 anos relatando esse consumo, demonstrando que as estratégias de prevenção devem começar aos 10 anos.

O II Levantamento Domiciliar sobre o Uso de Drogas Psicotrópicas no Brasil (2005) encontrou aumento na gravidade do uso/abuso/ dependência de substâncias, com 52,8\% dos meninos e 50,8\% das meninas entre 12 e 17 anos, relatando consumo de álcool na vida, com prevalência de dependência igual a $7 \%$.

É importante lembrar que o álcool e outras substâncias psicoativas podem ser utilizados por mulheres para automedicar a dor decorrente de situações de violência doméstica e trauma ${ }^{7,8}$. Para crianças e adolescentes, a convivência em lares disfuncionais com maus-tratos físicos e psicológicos pode aumentar em até 19 vezes a probabilidade do abuso ou dependência do álcool ${ }^{1,9}$. As meninas parecem experimentar maior estresse nesses lares conflitivos.

O Instituto de Prevenção e Pesquisa em Álcool e outras Dependências (IPPAD) desenvolve projeto em andamento que investiga a prevalência do consumo de substâncias psicoativas entre adolescentes e sua correlação com traumas, em uma amostra total de 300 sujeitos. São adolescentes do ensino médio, de ambos os sexos, provenientes de escolas públicas de Porto Alegre e cadastrados para estágio em empresa, no Centro de Integração Empresa Escola (CIEE/RS).

O CIEE/RS oferece um curso de dois meses de preparação ao mercado de trabalho, a jovens em contexto de vulnerabilidade, no qual está inserido o projeto Eu me Cuido, de prevenção ao uso de drogas, desenvolvido pelo IPPAD em parceria com o CIEE. Este estudo é um recorte que objetiva investigar o consumo do álcool por adolescentes do sexo feminino, buscando ampliar o conhecimento de um subgrupo que, apesar da grande vulnerabilidade, dispõe de escassez de pesquisas em nosso meio.

Os estudantes respondem, entre outros instrumentos, ao $\mathrm{Al}$ cohol Smoking Substance Involvement Screening Test (ASSIST) ${ }^{10}$, e resultados preliminares com 81 sujeitos (61 meninas e 20 meninos), com idades entre 15 e 24 anos e idade média de 17 anos (+/- 2,27), demonstram que $72,5 \%$ já consumiram bebidas alcoólicas.

Entre as meninas, 78,7\% têm entre 16 e 18 anos e $85,2 \%$ possuem o ensino médio incompleto; $87 \%$ são solteiras e $20 \%$ referem renda familiar mensal de R $\$ 1.500,00 ; 68,3 \%$ relatam uso de álcool na vida e $62 \%$, uso de álcool nos últimos três meses. Neste último período, $26,2 \%$ das adolescentes consumiram bebidas alcoólicas mensalmente e a metade delas, semanalmente.

As mais jovens referiram maior consumo de álcool, com 17,9\% das meninas entre 15 e 16 anos bebendo semanalmente, nos últimos três meses. Aquelas da faixa etária entre 17 e 18 anos relataram o maior consumo mensal (29,2\%), e as estudantes na faixa etária entre 19 e 20 anos relataram maior abstinência do álcool $(71,4 \%)$.

Não há diferença significativa, quanto ao consumo de álcool na vida, na comparação com os adolescentes do sexo masculino, embora o pequeno tamanho amostral possa interferir na ausência de significância. Para as meninas, há correlação entre o consumo de álcool na vida e renda familiar ( $\mathrm{p}=0,05 ; \mathrm{r}=0,39)$.

Os dados deste estudo são semelhantes aos de estudos epidemiológicos e outros que têm apontado o alto consumo de bebidas alcoólicas por adolescentes brasileiros, com diferenças regionais e destaque de maior consumo para a região Sul do país e a cidade de Porto Alegre ${ }^{5,6}$. O V Levantamento Nacional sobre o Consumo de Drogas Psicotrópicas ${ }^{5}$ encontrou prevalência de $71,0 \%$ para uso na vida, entre meninas do ensino fundamental e médio de escolas públicas de Porto Alegre.

Entre as hipóteses para o alto consumo de álcool entre os adolescentes gaúchos, está a forte tradição vitivinícola do estado, maior produtor do país, que pode estar favorecendo esse comportamento. No momento, o estado produz cerca de $80 \%$ do vinho brasileiro. No Rio Grande do Sul, a Lei no 11.705/2008, chamada de "Lei Seca", diferentemente de outros estados, tem sido pouco fiscalizada, o que, pensa-se, pode contribuir para o maior consumo, assim como para a provável tolerância da sociedade gaúcha. E, embora haja proibição legal para a venda de bebidas alcoólicas para menores de 18 anos, estas são livremente comercializadas no país.

Os agravos físicos e psicológicos que o consumo de álcool traz às adolescentes, destacando-se comportamento promíscuo, sexo sem proteção, doenças sexualmente transmissíveis (DST), abortos e traumas, justificam o rastreamento rotineiro desse comportamento de risco. Pesquisas sistemáticas são imperativas para o acompanhamento do consumo de substâncias psicoativas pelos adolescentes, por mulheres e, especialmente, entre as adolescentes. Por suas peculiaridades, estratégias específicas de prevenção para esse subgrupo são necessárias.

Pontifícia Universidade Católica do Rio Grande do Sul (PUCRS), Instituto de Prevenção e Pesquisa em Álcool e outras Dependências (IPPAD), Porto Alegre/RS. 
O pequeno tamanho amostral e a amostra de conveniência estão entre as limitações do presente estudo. São adolescentes de escolas públicas, do sexo feminino, em contexto de vulnerabilidade, que estão buscando inserir-se no mercado de trabalho. Mais estudos são necessários, com amostras maiores e de ambos os sexos. Mesmo assim, ele gera informações para um subgrupo do universo feminino que ainda carece de maior interesse investigativo no Brasil.

\section{Referências}

1. Guimarães ABP, Hochgraf PB, Brasiliano S, Ingberman YK. Aspectos familiares de meninas adolescentes dependentes de álcool e drogas. Rev Psiq Clín. 2009;36(2):69-74.

2. Bhuvaneswar CG, Chang G, Epstein LA, Stern TA. Alcohol use during pregnancy: prevalence and impact. J Clin Psychiatry. 2007;9(6):455-60.

3. Pinheiro SN, Laprega MR, Furtado EF. Morbidade psiquiátrica e uso de álcool em gestantes usuárias do Sistema Único de Saúde. Rev Saude Publica. 2005;39(4):593-8.

4. Cardoso LRD, Malbergier A, Figueiredo TFB. O consumo de álcool como fator de risco para a transmissão das DSTs/HIV/Aids. Rev Psiq Clín. 2008;35(Suppl. 1):70-5.
5. Galduróz JCF, Noto AR, Fonseca AM, Carlini EA. V Levantamento Nacional sobre o Consumo de Drogas Psicotrópicas entre Estudantes do Ensino Fundamental e Médio da Rede Pública de Ensino nas 27 Capitais Brasileiras. São Paulo: Centro Brasileiro de Informações sobre Drogas Psicotrópicas (CEBRID)/Secretaria Nacional Antidrogas (SENAD); 2004.

6. Carlini EA, Galduróz JCF, et al. II Levantamento Domiciliar sobre o Uso de Drogas no Brasil: estudo envolvendo as 108 maiores cidades do Brasil - 2005. Centro Brasileiro de Informações sobre Drogas Psicotrópicas - Universidade Federal de São Paulo (Unifesp) e Secretaria Nacional Antidrogas (SENAD), Gabinete de Segurança Institucional, Presidência da República.

7. Dunnegan SW. Violence, trauma and substance abuse. J Psychoactive Drugs. 1997;29(4):345-51.

8. Wilke ME, Fensterseifer DP, Pulcherio G. O estresse traumático da violência doméstica. Rev AMRIGS. 2007;51(2):149.

9. Danielson CK, Amstadter AB, Dangelmaier RED, Resnick HS, Saunders BE, Kilpatrick DG. Trauma-related risk factors for substance abuse among male versus female young adults. Addict Behav. 2009;34(4):395-9.

10. Henrique IFS, Micheli D, Lacerda RB, Formigoni MLOS. Validação da versão brasileira do teste de triagem do envolvimento com álcool, cigarro e outras substâncias (ASSIST). Rev Assoc Med Bras. 2004;50(2):199-206. 